Meta

Journal des tradlucteurs

Translators' Journal

\title{
Le bon sens
}

\section{Noëlle Guilloton}

Volume 24, numéro 4, décembre 1979

URI : https://id.erudit.org/iderudit/002989ar

DOI : https://doi.org/10.7202/002989ar

Aller au sommaire du numéro

Éditeur(s)

Les Presses de l'Université de Montréal

ISSN

0026-0452 (imprimé)

1492-1421 (numérique)

Découvrir la revue

Citer cet article

Guilloton, N. (1979). Le bon sens. Meta, 24(4), 471-477.

https://doi.org/10.7202/002989ar

Ce document est protégé par la loi sur le droit d'auteur. L'utilisation des services d'Érudit (y compris la reproduction) est assujettie à sa politique d'utilisation que vous pouvez consulter en ligne.

https://apropos.erudit.org/fr/usagers/politique-dutilisation/
Cet article est diffusé et préservé par Érudit.

Érudit est un consortium interuniversitaire sans but lucratif composé de l’Université de Montréal, l'Université Laval et l'Université du Québec à Montréal. Il a pour mission la promotion et la valorisation de la recherche. https://www.erudit.org/fr/ 


\section{LE BON SENS}

Chacun sait que, bien évidemment, il ne peut $\mathrm{y}$ avoir que deux sens à une rotation... oui, mais comment les nomme-t-on?

Étant donné la pluralité des mots et expressions qui désignent en français ces deux directions, il nous a semblé intéressant de les inventorier en explorant différents domaines scientifiques et techniques, depuis l'astronomie jusqu'à la robinetterie, en passant par la physique nucléaire et les sports.

Viennent immédiatement à l'esprit le sens des aiguilles d'une montre et le sens inverse des aiguilles d'une montre, tournures que consignent les ouvrages généraux. Mais ce n'est pas tout. 
- courant à droite courant à gauche 27

- rotation à droite rotation à gauche 28

- tournage à droite tournage à gauche 15

- filet(age) à droite filet(age) à gauche 15, 28

Certains documents précisent même :

- de gauche d̀ droite

de droite à gauche 3

Signalons également les termes dextrorsum 3,12 ou dextrorse 13 et senestrorsum 3,25 ou sinistrorsum 13 qui sont fréquemment donnés comme synonymes de formes plus \& courantes \$:

courant dextrorsum / syn. de courant dans le sens des aiguilles d'une montre

courant sinistrorsum / syn. de courant en sens inverse des aiguilles d'une montre 27

(il s'agit ici, bien sûr, du mouvement de l'eau de mer)

et sont aussi usités en télévision :

dextrorsum / clockwise

senestrorsum / counter-clockwise

ainsi que pour indiquer le sens de rotation des hélices :

- Si un observateur placé sur l'axe du cylindre voit l'hélice monter de sa droite vers sa gauche, l'hélice est dite directe ou sinistrorsum (à gauche); dans le cas contraire, elle est rétrograde ou dextrorsum (à droite) 》 13

L'hélice peut d'ailleurs être employée comme référence pour désigner un sens de rotation : hélice d̀ gauche

* Les vis ordinaires, les tire-bouchons ont leurs filets disposés suivant l'hélice à gauche $\$ 13$

Au passage, on aura relevé les adjectifs rétrograde et direct, qui semblent être consacrés en astronomie 4,19 et en techniques spatiales, disciplines auxquelles la mécanique les a sans doute empruntés.

Avant de clore ce tour d'horizon, il est bon de signaler certaines contradictions.

Lorsqu'on parle du sens normal dans le fonctionnement des machines-outils, il peut s'agir du synonyme du sens inverse des aiguilles d'une montre. 23 ... tandis qu'en robinetterie, on le donne comme équivalent du sens d'horloge :

* Le sens de fermeture d'un robinet est «sens normal ». Lorsque la fermeture s'obtient par rotation sens d'horloge de l'organe de commande vue de son extrémité de manceuvre $>2$ 
Il en va de même pour le sens positif que l'on définit tantôt comme équivalent du sens des aiguilles d'une montre, tantôt comme son contraire :

L'édition 1977 du petit Robert, par exemple, donne sens positif comme synonyme de sens direct ou sens trigonométrique, c'est-à-dire à gauche ou inverse des aiguilles d'une montre; de même, le Lexis distingue les deux mouvements de rotation autour d'un axe de la façon suivante :

« de droite à gauche (sens positif ou direct)

ou de gauche à droite (sens négatif ou rétrograde) » 19

Par contre, le Vocabulaire nucléaire de l'Hydro-Québec 16 établit l'équivalence suivante :

clockwise : dans le sens des aiguilles d'une montre, sens positif.

counter-clockwise / anti-clockwise : dans le sens opposé aux aiguilles d'une montre, dans le sens inverse des aiguilles d'une montre, sens négatif, inverse.

Cette équivalence est attestẹe également par le Dictionnaire français-anglais de l'électronique, physique nucléaire et sciences connexes 18 qui donne :

\section{anti-clockwise : sens négatif}

Il n'est certes pas aisé de tirer cette question au clair, et la prudence recommande évidemment au profane de s'en tenir aux expressions attestées hors de tout doute.

Pour terminer cet inventaire des expressions ou qualificatifs en usage dans divers domaines scientifiques et techniques, signalons en outre :

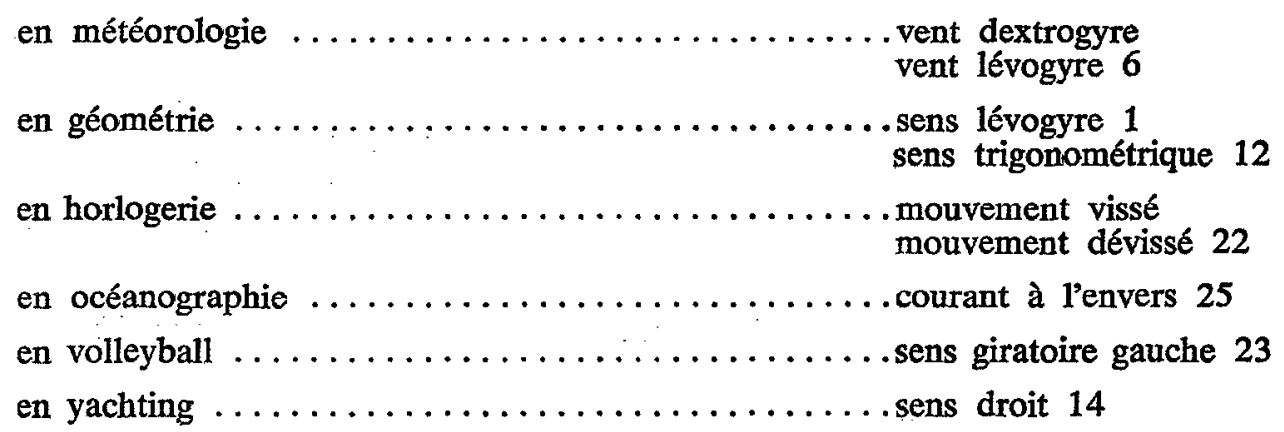

En conclusion, il semble permis d'nffirmer que, quel que soit le domaine, aussi technique ou scientifique qu'il puisse être, les expressions sens des aiguilles d'une montre et sens inverse des aiguilles d'une montre ou (de gauche) à droite et (de droite) à gauche sont correctes et comprises dans la plupart des contextes, tandis que sens horaire et sens antihoraire sont acceptables.

Toutefois, il est utile de noter qu'étant donné l'existence dans certaines disciplines d'expressions consacrées, qui coexistent d'ailleurs avec les formulations plus courantes données ci-dessus, il sera sans doute préférable d'employer, le cas échéant, l'expression ou le qualificatif propre à la langue technique en question (voir tableau récapitulatif). 
... À moins que l'on ne préfère écrire, avec Apollinaire : "Les aiguilles de l'horloge du quartier juif vont à rebours $\$ . . *^{*}$ Mais, hélas, n'est pas poète qui velut...

NOËLLE GUILLOTON

\section{BIBLIOGRAPHIE}

1. AUGER, Pierre, et autres, Encyclopédie internationale des sciences et des techniques, Paris, Presses de la Cité, 1969, t. 3, p. 713-714.

2. ASSOCIATION FRANÇAISE DE NORMALISATION (AFNOR), Sens de fermeture des robinets, NF E29-003, juin 1969.

3. BACHY, Françoise, Terminologie de la télévision, français-anglais, Louvain, Université catholique de Louvain, 1971, p. v.

4. BUREAU DES LONGITUDES, Annuaire pour l'an 1964, Paris, Gauthier-Villars et Cle, 1964 , p. 202.

5. CARNELUTTI, Daniele, Dictionnaire technique de l'automobile systématique illustré; Milan, Edizione Dizionario technico dell'automobile, c1963, p. 67 et 441.

6. COMMISSION DES COMMUNAUTES EUROPEENNES, BUREAU DE TERMINOLOGIE DE BRUXELLES, Bulletin de la traduction, no 49 , mai 1972, n.p.

7. COMMISSION ÉLECTROTECHNIQUE INTERNATIONALE, Index général du Vocabulaire électrotechnique international, Genève, Bureau central de la C.E.I., c1975, p. 430 et 450 .

8. COMMISSION ELECTROTECHNIQUE INTERNATIONALE, Vocabulaire électrotechnique international, $2^{\mathrm{e}}$ édition, Groupe 60, Radiocommunications, Genève, Bureau central de la C.E.I., 1970, p. 31.

9. COMPAGNIE GENÉRALE ELLCTRIQUE DU CANADA, Lexique anglais-français des interrupteurs de position, Montréal, 1977, p. 3 et 4.

10. COMPAGNIE GENÉRALE ÉLECTRIQUE DU CANADA, Lexique anglais-français des transformateurs de puissance, Montréal, s.d., p. 4, 6 et 7.

11. CUSSET, Francis, Vocabulaire technique, électricité, mécanique (etc.), 8e édition, Paris, Berger-Levrault, 1968, p. 396.

12. Dictionnaire des sciences, Paris, Seghers, c1962, p. 99 et 270.

13. Grand Larousse encyclopédique, 10 vol., Paris, Larousse, c1960-1964, aux entrées hélice et sens.

14. HEPP, Terence, Sports Dictionary in seven languages, Berlin, Sportverlag, c1962, p. 786.

15. HYDRO-QUEBEC, Dictionnaire électrotechnique, 4e édition revue et corrigée, Montréal, 1971 , p. 4 et 7.

16. HYDRO-QUÉBEC, Vocabulaire nucléaire, s.1., 1967, p. 15 et 18.

1\%. KETTRIDGE, J.O., French-English and English-French Dictionary of technical terms and phrases [....], London, Routledge \& Kegan Paul Ltd., 1967, vol. II, p. 635 et 658.

18. KING, G.G., Dictionnaire français-anglais, électronique, physique nucléaire et sciences connexes, Paris, Dunod, 1961, p. 334.

19. Lexis, Larousse de la langue française, Paris, Larousse, 1977, 2013 p.

20. MULLER, Paul, Dictionnaire de l'astronomie, Paris, Larousse, c1966, p. 77 et 207.

21. OFFICE DE LA LANGUE FRANÇAISE, Lexique anglais-français de l'électronique au Québec, Québec, Gouvernement du Québec, 1974, (Cahiers de l'Office de la langue française $n^{\circ} 22$ ), p. 37 et 43.

* Apollinaire, Guillaume, «Zone », Alcools, Paris, Gallimard, c1920. 
22. PESSIS-PASTERNAK, Guitta, Dictionnaire de l'audiovisuel français-anglais et anglaisfrançais, Paris, Flammarion, c1976, p. 116.

23. PHILIPPE, Jean, Lexique trilingue de la machine-outil et de loutillage, Paris, Dunod, 1964, p. 154, 200, 210, 234 et 260.

24. RÉGIE DE LA LANGUE FRANÇAISE, Lexique du volley-ball, Québec, Editeur officiel du Québec, c1976, p. 21.

25. ROBERT, Paul, Dictionnaire alphabétique et analogique de la langue française, Paris, Société du nouveau Littré, 1977, 2171 p.

26. SUBE, Ralph, Dictionnaire technique, physique nucléaire et technique nucléaire, Paris, Dunod, c1962, p. 1149.

27. Terminology Bulletin, New York, United Nations Secretariat, no 265, mars 1971, p. 68 et 82 .

28. WÜSTER, Eugene, Dictionnaire multilingue de la machine-outil, Londres, Technical Press, c1967, p.v.

TABLEAU RECAPITULATIF

sens des aiguilles d'une montre

sens du mouvement des aiguilles d'une montre

sens d'horloge
en sens d'horlog
dans le sens d'h
sens horaire
à droite
de gauche à droite
dextrorsum
dextrorse
sens rétrograde
sens \& normal
sens \& négatif
sens \& positif

en sens d'horloge

dans le sens d'horloge

sens horaire

à droite

de gauche à droite

dextrorsum

dextrorse
sens rétrograde
sens \& normal
sens \& négatif
sens \& positif

\begin{tabular}{|c|c|c|}
\hline \multicolumn{3}{|c|}{ domaine } \\
\hline $\begin{array}{l}\text { sens inverse des } \\
\text { aiguilles d'une montre }\end{array}$ & divers & $21,23,26$ \\
\hline $\begin{array}{l}\text { sens opposé aux } \\
\text { aiguilles d'une montre }\end{array}$ & $"$ & 16,18 \\
\hline $\begin{array}{l}\text { sens opposé des } \\
\text { aiguilles d'une montre }\end{array}$ & $"$ & 23 \\
\hline $\begin{array}{l}\text { contre le sens des } \\
\text { aiguilles d'une montre }\end{array}$ & $"$ & 25 \\
\hline $\begin{array}{l}\text { sens inverse du mouvement des } \\
\text { aiguilles d'une montre }\end{array}$ & " & 17 \\
\hline $\begin{array}{l}\text { sens inverse de celui des } \\
\text { aiguilles d'une montre }\end{array}$ & $"$ & 11 \\
\hline & robinetterie & 2 \\
\hline & & 5 \\
\hline & machine-outil & 28 \\
\hline sens inverse d'horloge & & 18 \\
\hline sens antihoraire & électricité & $9,10,21$ \\
\hline à gauche & divers & 15,27 \\
\hline de droite à gauche & divers & 3,21 \\
\hline senestrorsum & électricité & \\
\hline & océanographie & $3,7,8,12$ \\
\hline & & 13 \\
\hline sinistrorsum & $\begin{array}{l}\text { électricité } \\
\text { océanographie }\end{array}$ & $7,8,27$ \\
\hline hélice à gauche & vis & 13 \\
\hline sens direct & astronomie & $4,13,19$ \\
\hline & robinetterie & 2 \\
\hline sens \& normal » & machine-outil & 23 \\
\hline sens \& positif » & divers & 19,25 \\
\hline & $\begin{array}{l}\text { physique } \\
\text { nucléaire }\end{array}$ & 16 \\
\hline
\end{tabular}


(sens) dextrogyre

sens \& négatif
sens trigonométrique
(sens) lévogyre
dévissé
sens giratoire gauche
sens inverse

16,18

géométrie $\quad 12,25$

météorologie géométrie 1

horlogerie $\quad 22$

vissé

volley-ball 24

sens droit 